\title{
SIMULAÇÃO COM JOGOS DE MONTAR: UM INSTRUMENTO DE ENSINO PARA O PLANEJAMENTO E PROGRAMAÇÃO DE OBRAS
}

Marcelo D. Depexe ${ }^{1}$

\begin{abstract}
Resumo: Simulações e jogos didáticos são instrumentos que podem auxiliar o processo de ensino de diversas áreas da engenharia. O presente trabalho tem como objetivo discutir a utilização de jogos como instrumento de ensino para alunos de engenharia, em disciplinas de planejamento e programação de obras. Para isso, foi realizada uma simulação de planejamento e construção de um conjunto de 16 casas com jogos de montar. Inicialmente, os alunos elaboram o planejamento utilizando a técnica da Linha da Balanço. A fase seguinte é a montagem das casas, com controle do tempo de execução de cada etapa, para confronto em tempo real com a programação. Caso ocorram desvios em relação ao planejamento, os alunos devem tomar decisões no sentido de corrigir os desvios e finalizar o projeto dentro do prazo estipulado inicialmente. Observa-se que a utilização de jogos é bem aceita pelos alunos de engenharia, que apresentam maior interesse na aula, bem como nos assuntos debatidos. A realização da simulação possibilitou uma melhor compreensão de conceitos, bem como a possibilidade de utilização prática de tais conceitos, em uma situação que simula a realidade de forma simplificada. Conclui-se que, apesar de ainda pouco utilizados no processo de ensino superior, os jogos e simulações são uma importante ferramenta para o ensino de engenharia, pois facilitam a assimilação de determinados conceitos e permitem ao aluno a possibilidade de aplicação prática da teoria.
\end{abstract}

Palavras-chave: simulação, jogos, aprendizado, linha de balanço.

\begin{abstract}
Simulations and educational games are tools that can aid the process of teaching various areas of engineering. This paper aims to discuss the use of games as a teaching tool for engineering students in disciplines of planning and programming of works. For this, we performed a simulation of planning and construction of a set of 16 houses with a mounting game. Initially, students draw up a schedule using the technique of the Line of Balance. The next phase is the assembly of the houses, controling the execution time of each stage for real time confrontation with programming. In case of deviations from the plan, students must make decisions to correct deviations and complete the project within the initially stipulated time. It is observed that the use of games is well accepted by students of engineering, with the greatest interest in the classroom, as well as the issues discussed. The simulation provided a better understanding of concepts, as well as the possibility of practical use of such concepts in a situation that simulates the reality of a simpler way. We conclude that, although still little used in the process of higher education, games and simulations are an important tool for engineering education, because they facilitate the assimilation of certain concepts and allow students the possibility of practical application of theory.
\end{abstract}

Keywords: simulation, game, learning, line of balance.

1 UFSC - Universidade Federal de Santa Catarina, Programa de Pós-Graduação em Engenharia de Produção e-mail: marcelodepexe@yahoo.com.br 


\section{INTRODUÇÃO}

Simulações e jogos didáticos têm sido utilizados como instrumento de ensino em diversas áreas do conhecimento, como alternativa e complemento às aulas expositivas e de memorização. Desde o século XIX, a filosofia e pedagogia consideram que os jogos desempenham uma função biológica de adestrar para as atividades vitais. A pedagogia moderna e contemporânea atribuiu ao ludismo um caráter privilegiado de condição ou instrumento da formação humana básica (ABBAGNANO, 2007).

Conforme Ammar e Wright (1999), o uso de jogos didáticos facilita o aprendizado e aumenta consideravelmente o interesse e envolvimento dos alunos sobre o objeto de estudo. O aspecto lúdico dos jogos propicia satisfação e maior interesse nos alunos, além de ser uma ferramenta de fácil utilização e contextualização (TAO, CHENG e SUN, 2009).

Assim, os jogos e simulações são ferramentas pedagógicas eficientes para o ensino, uma vez que permitem aplicar seus conhecimentos na tomada de decisão, explorar estratégias alternativas e suas consequências, em um ambiente seguro e ao mesmo tempo próximo do real, de acordo com Walters, Coalter e Rasheed (1997) e Neill (2009).

Os jogos e simulações também podem ser utilizados como instrumentos de auxílio em treinamentos, de modo a formar as competências necessárias onde o conhecimento atual não é suficiente (RAUCH-GEELHAAR, JENKE e THURNES, 2003). Os autores salientam que apenas a combinação de diferentes formas de ensino, métodos e ferramentas é que possibilita a aquisição de competências. Assim, tais ferramentas de treinamento devem ser adaptadas de acordo com a demanda específica de conhecimento.

Desta forma, o presente trabalho tem como objetivo discutir a utilização de jogos como instrumento de ensino para alunos de engenharia, em disciplinas de planejamento e programação de obras, a partir da montagem de casas em miniatura.

\section{REVISÃO BIBLIOGRÁFICA}

A literatura apresenta diversos casos de aplicação de jogos e simulações como instrumentos de ensino, nas mais variadas áreas do conhecimento. Por exemplo, na área da saúde, Torkar, Pintarič e Koch (2010) apresentam um jogo de cartas utilizado para ensinar a importância do consumo de frutas e vegetais para a nutrição e saúde humana.

Sabri et al. (2010) apresentam um jogo computacional em terceira dimensão no qual alunos de medicina simulam uma cirurgia de artroplastia total do joelho (substituição da articulação do joelho por metal e polietileno). Os alunos podem simular todos os procedimentos cirúrgicos, se familiarizar com os instrumentos e tomar decisões em casos não previstos.

Também existem vários exemplos na área de administração e engenharia. Cano e Sáenz (2003) descrevem um laboratório de simulação de gestão de projetos, onde se procura estudar mecanismos de aprendizagem no ambiente de gestão de projetos. Os autores apresentam dois jogos computacionais, a partir dos quais analisam o impacto de fatores situacionais e motivação no aprendizado. Os jogos expõem os alunos a situações de planejamento e desenvolvimento de projetos.

Massukado e Schalch (2007) apresentam um software como recurso didático para a disciplina de Gestão de Resíduos Sólidos. Os autores afirmam que as simulações realizadas no software representam uma eficiente ferramenta do processo de construção do conhecimento, pois facilitou a compreensão das variáveis para se calcular a vida útil do aterro sanitário, ao invés de terem de memorizar uma lista de requisitos necessários para efetuar o mesmo cálculo. Além disso, as simulações possibilitaram que os alunos desenvolvessem um contato maior com o processo de tomada de decisão, contribuindo para o fortalecimento do aprendizado com autonomia, ou seja, do "aprender a aprender".

Souza e Silva et al. (2003) apresentam uma simulação de produção de canetas coloridas por profissionais de engenharia participantes de programas de pós-graduação. A simulação avalia o sistema de produção puxado e o sistema de produção empurrado. Através de várias simulações com diferentes estratégias, os profissionais produziram 60 canetas, com três variedades de cores, com o objetivo de questionar atitudes referentes à administração de estoques, definição de lotes de produção, sequenciamento das operaçôes, distribuição de equipes e layout do posto de trabalho. Os autores concluem que o sistema puxado apresenta melhores resultados por imobilizar menos capital, embora exija um planejamento mais mi- 
nucioso, com a utilização de gargalos e controle de estoques, de modo a atender às necessidades variáveis dos clientes.

Sun (1998) apresenta um jogo que simula um sistema de produção de veículos. O objetivo do jogo é explorar os conceitos de just-in-time (JIT) e material requeriment planning (MRP) por meio de uma simulação. $\mathrm{O}$ autor utiliza apenas papel e cartas de baralho para representar os carros e o sistema kanban utilizado na linha de montagem. Outro exemplo da utilização de jogos para ensino de conceitos de sistemas de produção é apresentado por Dorneles et al. (2006), que relata uma experiência na qual se utiliza um jogo para complementar o processo de aprendizado em sala de aula. A partir do jogo realizado com blocos de montar tipo Lego ${ }^{\circledR}$, os alunos puderam compreender melhor os conceitos da produção enxuta. Os autores salientam que uma das vantagens dos jogos é a possibilidade de comparação entre situações diferenciadas de layout de trabalho, com rapidez e baixo custo.

Os blocos de montar também são utilizados em treinamento de executivos, conforme apresentado por Roos, Victor e Statler (2004), para o desenvolvimento de novas estratégias. Segundo os autores, esta é uma forma de ativar a imaginação, integrar as dimensões de experiência cognitiva, social e emocional. Os participantes utilizam os blocos para representar e discutir questôes estratégicas, organizacionais e ambientais, de modo a gerar novas idéias a respeito dos diferentes elementos do conteúdo das estratégias. De maneira similar, Bürgi, Victor e Lentz (2004) também apresentam o uso dos blocos de montar para o desenvolvimento de estratégias em uma empresa do setor químico. Este método auxilia os gerentes a conhecer melhor seu negócio, de modo a preparalos para reagir a mudanças repentinas.

$\mathrm{Na}$ área de gerenciamento da construção civil, as simulações são utilizadas por diversos pesquisadores em situações nas quais não se pode avaliar um evento real, devido ao longo período de tempo necessário. Tommelein, Riley e Howell (1998) apresentam a simulação de um jogo sobre a utilização de tempos de folga na programação. Os autores demonstram que é possível reduzir o desperdício e do tempo de duração do projeto ao se reduzir a variabilidade do fluxo de trabalho.

Vargas et al. (1998b) descrevem uma aplicação de jogos para a simulação da construção de obras prediais. A simulação consta da execução de todas as etapas necessárias para a construção de banheiros sociais de um edifício de dez pavimentos em modelo reduzido confeccionado em cartolina. A execução foi planejada com auxílio da técnica da Linha de Balanço. O objetivo da simulação é aplicar as técnicas de avaliação de produtividade e medição de perdas, além de evidenciar a aplicação de técnicas modernas de gerenciamento de obras.

Vargas et al. (1998a) apresentam melhorias no modelo anterior, com a simulação da construção de um conjunto de casas em escala reduzido, confeccionadas em cartolina colorida. A simulação procura demonstrar as vantagens da utilização da técnica da Linha de Balanço. Para isso, os autores apresentam dois exercícios. No primeiro, os participantes executam todas as etapas do edifício, sem qualquer planejamento. No segundo exercício, utiliza-se o dimensionamento das equipes segundo a técnica da Linha de Balanço, o que acarreta menor variabilidade e aumento da produtividade, devido à redução dos tempos improdutivos e auxiliares.

Saito et al. (2000) e Santos et al. (2002) apresentam um jogo que simula a construção de um edifício de dez pavimentos que permite avaliar fatores como velocidade, qualidade e desperdícios. Além de abordar a técnica de programação da Linha de Balanço, o jogo permite a avaliação de elementos como a compatibilização de projetos, construtibilidade, estratégias de mercado, trabalho em equipe e estratégia de produção. Santos et al. (2002) afirmam que a competitividade que surge entre os grupos é um fator estimulante para a conclusão e compreensão dos conceitos. As equipes se comportam como se fossem empresas reais, com mudanças de estratégia em função dos concorrentes que se encontram em posição mais vantajosa.

Saffaro et al. (2003) apresentam um jogo de montagem de casas do tipo Lego ${ }^{\circledR}$ com o objetivo de avaliar o comportamento dos jogadores com relação aos princípios da Lean Production. O jogo consiste na montagem de 15 casas e a medida do desempenho de cada equipe. Com isso, o jogo permite discussões relativas à administração de estoques de matéria prima, trabalho em progresso, sequencia de tarefas, balanceamento de pacotes de trabalho e definição de células de trabalho.

Portanto, os jogos e simulações são utilizados para o ensino de diversas áreas do conhe- 
cimento, tanto sob a forma de jogos com peças físicas quanto em jogos computacionais. Hunecker (2009) salienta que jogos computacionais desenvolvidos especificamente para o ensino de determinado assunto geralmente demandam longos períodos de desenvolvimento, além de elevado custo. Uma alternativa apontada é a adaptação de jogos comerciais já existentes, nos quais pode-se abordar temas como estratégia, logística e utilização de recursos.

Por outro lado, a utilização de jogos com peças físicas apresenta custo bem menor, se comparado ao desenvolvimento de softwares específicos. Desta forma, o presente trabalho apresenta o relato da utilização de blocos de montar para a simulação da programação e construção de um conjunto habitacional composto por 16 casas, conforme método descrito a seguir.

\section{MÉTODO DE TRABALHO}

A simulação em questão consiste no planejamento e construção de um conjunto de 16 casas com jogos de montar do tipo Lego System ${ }^{\circledR}$. O objetivo do experimento é explorar os conceitos de programação e controle de obras, principalmente o conceito da Linha de Balanço, em uma situação prática que simule a realidade. A utilização de tal jogo é adequada para a realização desta simulação, por permitir a execução de um grande número de repetições em um curto espaço de tempo, uma vez que trabalhamos com casas em pequena escala.

Para a elaboração da programação com a técnica da Linha de Balanço, é necessário definir qual a unidade básica de repetição, qual o tempo para sua execução e a mão-de-obra necessária. Conforme Maziero (1990), unidade básica é o tipo de unidade que compóe o projeto e deve ser repetida até a sua conclusão. As partes de uma edificação como o pavimento, apartamento ou bloco de apartamentos, que reproduzam um conjunto de operaçôes a serem repetidas em um projeto determinam a unidade básica, sendo que esta divisão depende do tipo e dimensão do projeto. Neste caso, a unidade básica de repetição será uma casa.

A seguir, deve-se definir as etapas da construção que serão objeto de controle e planejamento, bem como os respectivos tempos para sua execução. Com estas informações, pode-se elaborar a programação com a Linha de Balanço. A fase seguinte é a montagem das casas, com controle do tempo de execução de cada etapa, para confronto em tempo real com a programação. Caso se verifiquem desvios em relação ao planejamento, os alunos podem tomar decisões no sentido de corrigir os desvios e finalizar o conjunto das 16 casas dentro do prazo estipulado inicialmente. A seguir, descreve-se a simulação e resultados obtidos.

\section{SIMULAÇÃO}

A simulação da programação e construção de um conjunto de 16 casas foi realizada com o auxílio do jogo de peças de montar. Um exemplo de casa montado na simulação pode ser visualizado na Figura 1.

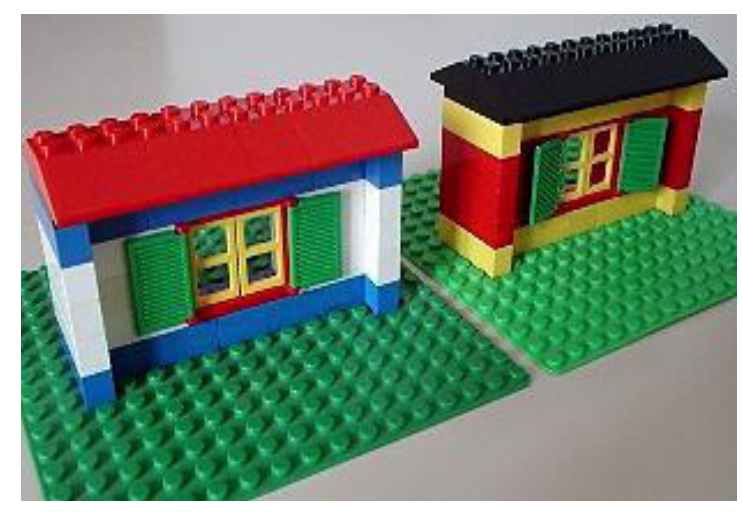

Figura 1 - Exemplo das casas montadas

Inicialmente, é necessário que se realize a programação das atividades a serem realizadas. Para isso, determina-se o número de peças da casa padrão e o tempo necessário para a montagem de cada peça.

A casa foi então divida nas seguintes etapas:

- fundação - 6 peças;

- alvenaria principal - 9 peças;

- alvenaria lateral - 6 peças;

- cinta de amarração - 5 peças;

- laje - 2 peças;

- telhado - 9 peças.

Após a realização de um teste de montagem, adotou-se que o índice de produtividade a ser utilizado seria de dois segundos por peça. Cabe salientar que, como se trata de uma simulação, as unidades de medida devem ser adaptadas para refletirem a realidade do experimento executado, ou seja, ao invés de utilizar unidades habituais da construção civil, como dias, horas e metro quadrado, utiliza-se aqui o segundo e a peça como medida unitária. 
Uma vez definidas as etapas e o índice de produtividade, realiza-se a programação utilizando a técnica da Linha de Balanço. Nesta fase, determinou-se que os serviços correspondentes à alvenaria principal e ao telhado seriam realizados por dois funcionários simultaneamente, por serem compostos por um maior número de peças, enquanto os demais serviços seriam executados por um só funcionário. $\mathrm{O}$ diagrama com a Linha de Balanço pode ser visualizado na Figura 2.

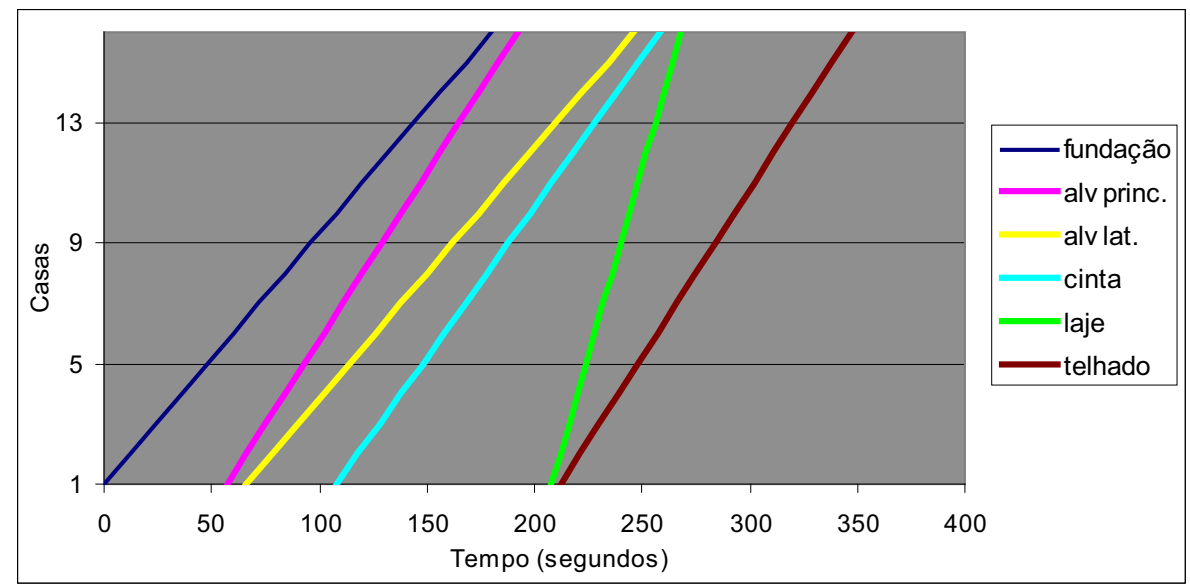

Figura 2 - Programação em Linha de Balanço

Devido à velocidade de execução do experimento, as medições para conferência do índice de produtividade real foram realizadas em conjuntos de quatro casas, para cada serviço separadamente.

Deste modo, após a realização completa de um serviço nas quatro primeiras casas, verifica-se o tempo gasto nessa operação, de modo a obter simultaneamente o gráfico do serviço executado até o momento, o índice de produtividade, o tempo médio por casa e o número de funcionários necessários para realizar a atividade utilizando o novo índice de produtividade.

Com base nessas informações, pode realizar intervenções para que os prazos finais de execução de cada serviço sejam cumpridos. A

Tabela 1 apresenta o resultado da simulação e a Figura 3 apresenta o gráfico com as atividades programadas e executadas (assinaladas com ${ }^{*}$ ).

Tabela 1 - Resultado da simulação

\begin{tabular}{|c|c|c|c|c|c|}
\hline & & casas $1-4$ & casas $5-8$ & casas 9-12 & casas $13-16$ \\
\hline \multirow{5}{*}{ fundação } & tempo total (s) & 48,8 & 51,9 & 46,9 & 56,2 \\
\hline & tempo/casa (s) & 12 & 13 & 12 & 14 \\
\hline & $n^{\circ}$ funcionários & 1 & 1 & 1 & 1 \\
\hline & produtiv. (s/peça) & 2,0 & 2,2 & 2,0 & 2,3 \\
\hline & nova equipe & 1 & 1 & 1 & 1 \\
\hline \multirow{5}{*}{ alv princ. } & tempo total & 48,1 & 48,7 & 31,7 & 20,3 \\
\hline & tempo/casa & 12 & 12 & 8 & 5 \\
\hline & $\mathrm{n}^{\circ}$ funcionários & 2 & 2 & 3 & 4 \\
\hline & produtiv. (s/peça) & 2,7 & 2,7 & 2,6 & 2,3 \\
\hline & nova equipe & 3 & 3 & 3 & 2 \\
\hline \multirow{5}{*}{ alv lat. } & tempo total & 70,0 & 51,8 & 26,7 & 23,3 \\
\hline & tempo/casa & 18 & 13 & 7 & 6 \\
\hline & $\mathrm{n}^{\mathrm{o}}$ funcionários & 1 & 1 & 2 & 2 \\
\hline & produtiv. (s/peça) & 2,9 & 2,2 & 2,2 & 1,9 \\
\hline & nova equipe & 1 & 1 & 1 & 1 \\
\hline
\end{tabular}




\begin{tabular}{|c|c|c|c|c|c|}
\hline \multirow{5}{*}{ cinta } & tempo total & 47,6 & 51,8 & 20,8 & 53,1 \\
\hline & tempo/casa & 12 & 13 & 5 & 13 \\
\hline & $n^{\circ}$ funcionários & 1 & 1 & 2 & 1 \\
\hline & produtiv. (s/peça) & 2,4 & 2,6 & 2,1 & 2,7 \\
\hline & nova equipe & 1 & 1 & 1 & 1 \\
\hline \multirow{5}{*}{ laje } & tempo total & 24,3 & 12,7 & 23,7 & 19,3 \\
\hline & tempo/casa & 6 & 3 & 6 & 5 \\
\hline & $\mathrm{n}^{\circ}$ funcionários & 1 & 2 & 1 & 1 \\
\hline & produtiv. (s/peça) & 3,0 & 3,2 & 3,0 & 2,4 \\
\hline & nova equipe & 2 & 2 & 1 & 1 \\
\hline \multirow{5}{*}{ telhado } & tempo total & 50,1 & 23,7 & 38,4 & 34,2 \\
\hline & tempo/casa & 13 & 6 & 10 & 9 \\
\hline & $\mathrm{n}^{\circ}$ funcionários & 2 & 3 & 2 & 2 \\
\hline & produtiv. (s/peça) & 2,8 & 2,0 & 2,1 & 1,9 \\
\hline & nova equipe & 3 & 2 & 2 & 2 \\
\hline
\end{tabular}

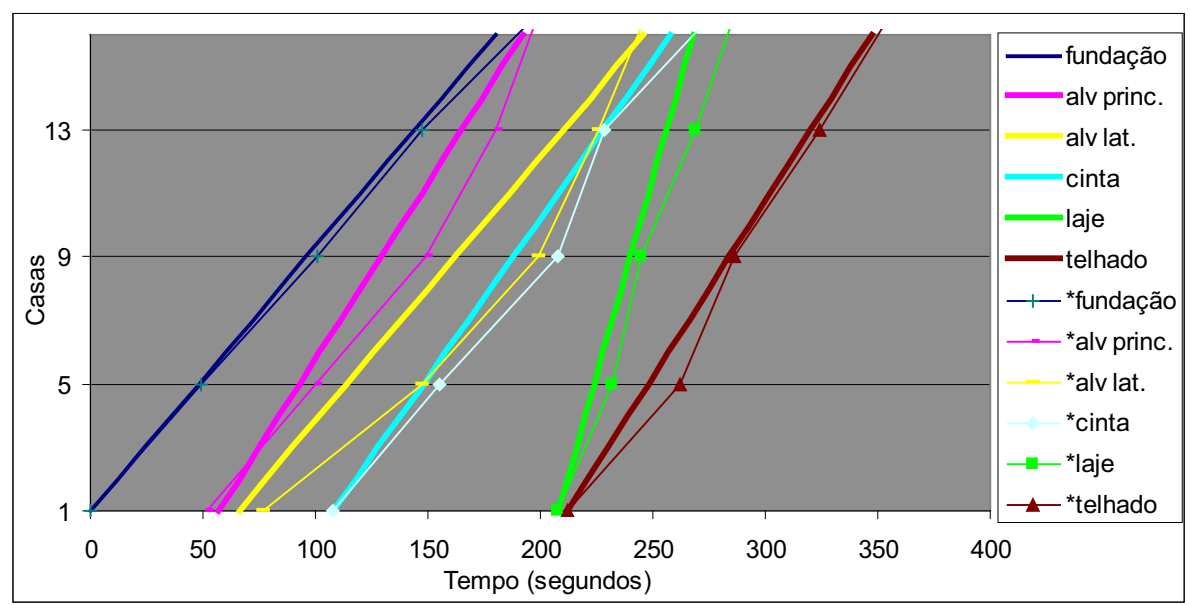

Figura 3 - Atividades programadas e executadas

A primeira etapa, correspondente a fundação, transcorre sem problemas. Apenas nas últimas quatro casas é que se verifica um atraso em relação à programação. A alvenaria principal, composta por nove peças, foi executada com dois funcionários trabalhando simultaneamente e foi iniciada alguns segundos antes do previsto. Entretanto, ao invés das quatro casas serem executadas em 36 segundos, levou-se 48,1 segundos. A planilha indica, assim, a necessidade de utilizar mais um funcionário. No entanto, optou-se por manter os dois funcionários na execução das próximas quatro casas, que foram executadas em 48,7 segundos, ou seja, praticamente o mesmo desempenho verificado anteriormente.

Para a execução da alvenaria principal das casas 9 a 13, adicionou-se mais um funcionário, que permitiu a redução do tempo para 31,7 se- gundos, mas ainda não é o suficiente para atingir o planejamento, devido ao atraso ocorrido nas oito casas anteriores. Deste modo, foi necessário o emprego de quatro funcionários para que o prazo fosse cumprido. As demais etapas seguem o mesmo procedimento.

A partir do experimento, os índices médios de produtividade para cada etapa são os seguintes:

- fundação - 2,1 segundo/peça;

- alvenaria principal - 2,6 segundo/peça;

- alvenaria lateral - 2,3 segundo/peça;

- cinta de amarração - 2,4 segundo/peça;

- laje - 2,9 segundo/peça;

- telhado - 2,2 segundo/peça.

Assim como ocorre em situações reais, observam-se variações no índice de produtividade das diferentes atividades, o que mostra ser equivocada a adoção de um único índice para todos os 
serviços. Isso se deve ao fato de que, apesar de se tratar de peças semelhantes, existem diferenças no nível de dificuldade de fixação para cada serviço.

Além disso, ao se executarem diversas casas, os funcionários necessitam passar de uma casa para a outra, o que gasta um tempo que é computado, mas que não agrega valor. No caso da laje, que apresentou o maior índice (2,9 segundos/peça) e possui o menor número de peças (apenas duas), conclui-se que a maior parte do tempo gasto refere-se ao deslocamento do funcionário entre as casas (tempo não produtivo), o que eleva o tempo gasto por unidade de peça montada.

Assim, a realização do experimento proporcionou aos alunos a verificação prática de diversas situaçôes que ocorrem durante a execução e planejamento de obras.

\section{CONCLUSÕES}

O presente trabalho apresenta o relato de um experimento no qual se utiliza uma simulação para complementar o processo de aprendizado em sala de aula. Ao se analisar a bibliografia citada, observa-se uma maior incidência de jogos com peças físicas voltados para o ensino de engenharia, principalmente civil e de produção, enquanto outras áreas do conhecimento utilizam simulaçôes e jogos computacionais. Observa-se que o jogo proposto é adequado ao ensino de programação e planejamento de obras, pois reflete problemas similares aos reais, como atrasos no cronograma, falta de material, produtividade diferente entre os funcionários e até mesmo identificar falhas no planejamento.

A simulação possibilitou uma melhor compreensão de conceitos relacionados ao planejamento e programação de obras, bem como a possibilidade de utilização prática de tais conceitos, em uma situação que simula a realidade de forma simplificada. Desta forma, pode-se evidenciar aos alunos a importância do acompanhamento do avanço físico da obra em tempo real.

Uma vez que a programação da obra é realizada a partir de índices de produtividade, a verificação prática de tais índices durante o andamento da obra auxilia a tomada de decisão por parte do gerente da obra, com o objetivo de corrigir desvios quanto à programação em curtos espaços de tempo. Assim, há maiores possibilidades de que as atividades serão encerradas dentro do prazo previamente estabelecido.
Observa-se que a utilização de jogos é bem aceita pelos alunos de engenharia, que apresentam maior interesse na aula, bem como nos assuntos debatidos. Desta forma, um jogo pode simular situações similares à realidade, o que leva o aluno a procurar soluções utilizando os conceitos e teorias apresentados em aula, pois naquele momento o aluno assume a posição de um gerente industrial ou de um engenheiro de obras, que deve tomar decisões de modo a garantir a execução do planejamento.

Durante a simulação, pode-se explorar diversas idéias e possibilidades para a verificação do resultado e avaliação de outras alternativas, em um ambiente seguro e controlado, com pequena utilização de recursos financeiros e de tempo. Portanto, apesar de ainda pouco utilizados no processo de ensino superior, os jogos e simulações são uma importante ferramenta para o ensino de engenharia, pois facilitam a assimilação de determinados conceitos e permitem ao aluno a possibilidade de aplicação prática da teoria.

\section{REFERÊNCIAS}

ABBAGNANO, Nicola. Dicionário de Filosofia. 5a Ed. São Paulo: Martins Fontes, 2007, 1210 p.

AMMAR, Salwa; WRIGHT, Ronald. Experiential learning activities in operations management. International Transactions in Operational Research, v. 6, n. 2, p. 183-197, 1999.

BÜRGI, Peter; VICTOR, Bart; LENTZ, Jody. Modeling how their business really works prepares managers for sudden change. Strategy $\&$ Leadership, v. 32, n.2, p. 28-35, 2004.

CANO, J. L.; SÁENZ, M. J. Project management simulation laboratory: experimental learning and knowledge acquisition. Production Planning \& Control, v. 14, n. 2, p. 166-173, 2003.

DORNELES, J. B.; DEPEXE, M. D.; SILVEIRA, J. P.; GASPARETTO, F. C.; SANTOS, D. G.; HEINECK, Luiz F. M. Montagem de carrinhos - aprendizado de conceitos da construção enxuta por meio de jogos didáticos. In: XI ENCONTRO NACIONAL DE TECNOLOGIA DO AMBIENTE CONSTRUÍDO, 2006, Florianópolis. Anais..., 2006. 
HUNECKER, Felix. A generic process simulation-model for educational simulations and serious games. On The Horizon, v. 17, n. 4, p. 313322, 2009.

MASSUKADO, Luciana Miyoko; SCHALCH, Valdir. Simulação no ensino de engenharia - avaliando a aplicação do software SIMGERE sob o paradigma do "aprender a aprender". Revista de Ensino de Engenharia, v. 26, n. 2, p. 40-46, 2007.

MAZIERO, L.T.P. Aplicação do conceito do método da linha de balanço no planejamento de obras repetitivas. Um levantamento das decisóes fundamentais para sua aplicação. 1990. Dissertação (Mestrado em Engenharia) - Universidade Federal de Santa Catarina, Florianópolis, 1990.

NEILL, Tim. Serious games: learning for the igeneration. Development and Learning In Organizations, v. 23, n. 4, p. 12-15, 2009.

RAUCH-GEELHAAR， C.; JENKE， K.; THURNES, C. M. Gaming in industrial management - quality and competence in advanced training. Production Planning \& Control, v. 14, n. 2, p. 155-165, 2003.

ROOS, Johan; VICTOR, Bart; STATLER, Matt. Playing seriously with strategy. Long Range Planning, v. 37, p. 549-568, 2004.

SAFFARO, Fernanda Aranha; BRESSIANI, Lucia; SANTOS, Débora de Góis; HEINECK, Luiz Fernando. Discussão de princípios da lean production através de um jogo didático. In: SIMPÓSIO BRASILEIRO DE GESTÃO E ECONOMIA DA CONSTRUÇÃO, III, 2003, São Carlos, Anais... São Carlos - SP, 2003, 10 p.

SAITO, Ângela Mayumi; GONZALEZ, Edinaldo Favareto; BOTTER, Ricardo Roberto; BERNARDINELI, Rejane Lucia Rufo; SAPATA, Sonia Moreira Molina. Jogo de análise de gerenciamento de obras. In: ENCONTRO TECNOLÓGICO DA ENGENHARIA CIVIL E ARQUITETURA DE MARINGÁ, 2000, Maringá. Anais... Maringá - PR, 2000, 5p.

SANTOS, Débora de Góis; BORGES, Valeska Prada; PRADO, Renato Lucio; HEINECK, Luiz
Fernando M. O ensino de linha de balanço e variabilidade através de um jogo didático. In: ENCONTRO NACIONAL DE TECNOLOGIA DO AMBIENTE CONSTRUÍDO, IX, 2002, Foz do Iguaçu. Anais... Foz do Iguaçu - PR, 2002, p. 767-776.

SABRI, Hamed; COWAN, Brent; KAPRALOS, Bill; PORTE, Mark; BACKSTEIN, David, DUBROWSKIE, Adam. Serious games for knee replacement surgery procedure education and training. Procedia Social and Behavioral Sciences, v. 2, n. 2, p. 3483-3488, 2010.

SOUZA e SILVA, Maria de Fátima; BRESSIANI, Lucia; SAFFARO, Fernanda Aranha; SANTOS, Débora de Gois; HEINECK, Luiz Fernando Mählmann. Sistema de produção puxado e sistema de produção empurrado: simulação através de jogo didático de montagem de canetas, associando idéias e conceitos ao ambiente da construção civil. In: SIMPÓSIO BRASILEIRO DE GESTÃO E ECONOMIA DA CONSTRUÇÃO, III, 2003, São Carlos, Anais... São Carlos - SP, 2003, 11 p.

SUN, H. A game for the education and training of production/operations management. Education + Training, v. 40, n. 9, p. 411-416, 1998.

TAO, Yu-Hui; CHENG, Chieh-Jen; SUN, SzuYuan. What influences college students to continue using business simulation games? The Taiwan experience. Computers \& Education, v. 53, n. 3, p. 929-939, 2009.

TOMMELEIN, Iris D.; RILEY, David; HOWELL, Greg A. Parade Game: impact of work flow variability on succeeding trade performance. In: CONFERENCE OF THE INTERNATIONAL GROUP FOR LEAN CONSTRUCTION, 6., 1998, Guarujá. Proceedings... Guarujá, Brasil. 1998, 14p.

TORKAR, Gregor; PINTARI\% Miša; KOCH, Verena. Fruit and vegetable playing cards: utility of the game for nutrition education. Nutrition \& Food Science, v. 40, n. 1, p. 74-80, 2010.

VARGAS, Carlos Luciano Sant'Ana; KRÜGER, José A.; HEINECK, Luiz Fernando M.; COELHO, Renato de Q. Avaliação de produtividade 
e de perdas na construção civil - simulação utilizando modelo reduzido para demonstrar as vantagens do uso da Linha de Balanço na programação da obra e de inovações tecnológicas no canteiro. In: ENCONTRO NACIONAL DE TECNOLOGIA DO AMBIENTE CONSTRUÍDO, VII, 1998. Florianópolis. Anais... Florianópolis, 1998a, v. 2, p.159-168.

VARGAS, Carlos Luciano Sant'Ana; MENDES Jr., Ricardo; HEINECK, Luiz Fernando Mahlmann; KRÜGER, José Adelino. Programação e controle de atividades repetitivas na execução de obras com modelo reduzido utilizando a técnica da Linha de Balanço. In: ENCONTRO NACIONAL DE ENGENHARIA DE PRODUÇÃO, 18, 1998. Niterói. Anais... Niterói, 1998b, 8p.

WALTERS, B. A.; COALTER, T. M. \& RASHEED, A. M. A. Simulation games in business policy courses: is there value for students? Journal of Education for Business, v. 72, n. 3, p. 170-174, 1997. 